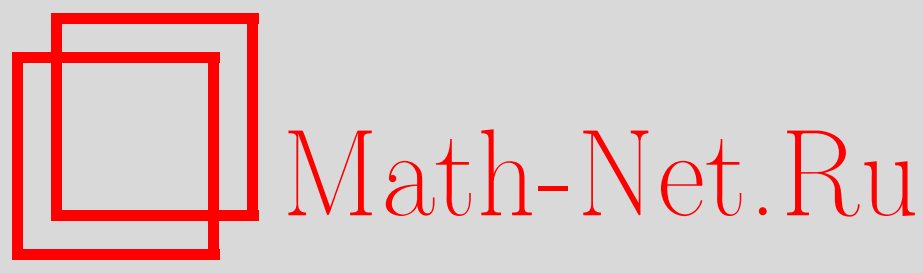

В. Н. Голощапов, П. В. Ролдугин, О свойствах булевых функций, не имеющих имплицент от трех переменных, Дискрет. матем., 2014, том 26, выпуск 2, 25-41

DOI: https://doi.org/10.4213/dm1278

Использование Общероссийского математического портала Math-Net.Ru подразумевает, что вы прочитали и согласны с пользовательским соглашением http://www . mathnet.ru/rus/agreement

Параметры загрузки :

IP: 44.207 .124 .84

26 апреля 2023 г., 14:23:09

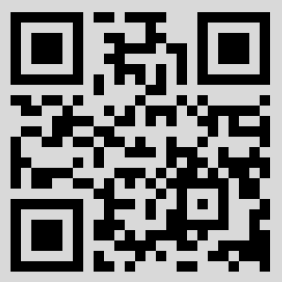




\title{
О свойствах булевых функций, не имеющих имплицент от трех переменных
}

\author{
() 2014 г. В. Н. Голощапов*, П. В. Ролдугин ${ }^{\dagger}$
}

Пусть $F_{n}-$ множество всех булевых функций от $n$ переменных и $M_{n} \subset F_{n}-$ класс функций, не имеющих имплицент от трех или менее существенных переменных. Обозначим через $n_{\max }^{(3)}(m)$ наибольшее значение $n$, при котором класс $M_{n}$ содержит функцию веса $m$. В статье показано, что $n_{\max }^{(3)}(11)=5$, $n_{\max }^{(3)}(12)=11, n_{\max }^{(3)}(13)=11$ (ранее точные значения были известны только при $m \leqslant 10)$. На основе тензорного произведения матриц предложена бинарная операция $\xi: F_{n_{1}} \times F_{n_{2}} \rightarrow F_{n_{1} \cdot n_{2}}$ и доказано, что если один из аргументов операции $\xi$ лежит в $M_{n_{1}}$, а другой - такая булева функция $f\left(x_{1}, \ldots, x_{n_{2}}\right)$, что $f\left(x_{1}, \ldots, x_{n_{2}}\right) \vee f\left(\bar{x}_{1}, \ldots, \bar{x}_{n_{2}}\right)$ принадлежит $M_{n_{2}}$, то результат операции всегда лежит в $M_{n_{1} \cdot n_{2}}$. Показано, что при ослаблении указанных условий на аргументы операции $\xi$ результат не обязан лежать в $M_{n_{1} \cdot n_{2}}$. Операция $\xi$ используется в алгоритме построения булевых функций без имплицент от трех переменных со сколь угодно малым отношением веса функции к числу переменных.

Ключевые слова: булева функция, имплицента, комбинаторно полная матрица, комбинаторно достаточная матрица.

\section{Введение}

Будем использовать следующие обозначения и определения. Через $V_{n}$ обозначим множество двоичных векторов длины $n$. Для булевой функции $f$ от $n$ переменных обозначим через $E_{f}$ множество ее выполняющих векторов: $E_{f}=$ $\left\{\left(\alpha_{1}, \ldots, \alpha_{n}\right) \in V_{n}: f\left(\alpha_{1}, \ldots, \alpha_{n}\right)=1\right\}$; весом функции $f$ называется $\|f\|=\left|E_{f}\right|$. Длиной булевой функции назовем число ее существенных переменных. Имплицентой ([6]) булевой функции $f$ является такая не равная константе булева функция $g$, что $f \cdot g=f$ (что эквивалентно условию $E_{f} \subseteq E_{g}$ ).

Настоящая работа является продолжением работ [1] - [5] по исследованию булевых функций, не имеющих имплицент от фиксированного числа переменных. В различных прикладных областях возникает необходимость решения систем булевых нелинейных уравнений. При решении таких систем можно использовать имплиценты булевых функций как средство для перехода к системам-следствиям, которые, возможно, будут решаться менее трудоемким способом по сравнению с исходной системой. Функции, не имеющие имплицент от малого числа неизвестных, образуют класс, устойчивый к попытке свести систему уравнений, составленную из функций

* Место работы: Московский государственный технический университет радиотехники, электроники и автоматики, e-mail: pavroldugin@rambler.ru

${ }^{\dagger}$ Место работы: Лаборатория ТВП, e-mail: goloschapov@list.ru 
этого класса, к решению системы, в которой каждое уравнение зависит от небольшого числа переменных.

В [3] полностью описан класс булевых функций, не имеющих имплицент от двух переменных. В статье [4] получен ряд нижних оценок весов функций, не имеющих имплицент от $k$ переменных для произвольного $k \geqslant 3$. В [1] получены верхние оценки числа переменных булевых функций небольшого веса, не имеющих имплицент длины $k$.

Булева матрица $B$ размера $m \times n, n \geqslant k>1$, называется комбинаторно полной порядка $k([4])$, если в любой ее подматрице размера $m \times k$ для любого булева вектора $\vec{v} \in V_{k}$ найдется строка, равная $\vec{v}$. Иначе говоря, при выборе любых $k$ столбцов в матрице $B$ полученная подматрица должна содержать каждую из $2^{k}$ возможных строк (но не обязательно только по одному разу). Отметим, что перестановка строк или столбцов, инвертирование столбцов комбинаторной полной матрицы дают комбинаторно полные матрицы того же порядка и размера. Более подробно эти и другие элементарные свойства комбинаторно полных матриц рассмотрены в [1].

В [4] показано, что булева функция $f$ не имеет имплицент длины $k$ тогда и только тогда, когда матрица, строками которой являются все векторы, лежащие в $E_{f}$, комбинаторно полная порядка $k$. В указанной матрице число строк совпадает с весом функции $f$, а число столбцов - с количеством переменных функции $f$. В частности, в [4] доказано, что наибольшее количество $n_{\max }^{(k)}(m)$ столбцов комбинаторно полной матрицы порядка $k$ при фиксированном числе строк $m$ совпадает с наибольшим значением $n$, при котором найдется булева функция от $n$ переменных, обладающая весом $m$ и не имеющая имплицент от $k$ или менее переменных.

Основное внимание в настоящей работе уделено изучению случая комбинаторной полноты порядка 3. Найдены (утверждение 6) точные значения наибольшего количества столбцов комбинаторно полной матрицы порядка 3 с числом строк 11, 12 и 13 (ранее были известны значения только при числе строк, не больше 10 - см. [1]). Определено понятие комбинаторно достаточной матрицы порядка $k$, которое является ослабленным вариантом понятия комбинаторной полноты. Введена операция $T \otimes A \oplus \bar{T} \otimes \bar{A}$ между двоичными матрицами $A$ и $T$, результат которой является комбинаторно полной матрицей порядка 3 , если одна из матриц $A, T$ является комбинаторно полной, а другая комбинаторно достаточной матрицей порядка 3 (теорема 1 и следствие 2). Показано, что эти условия нельзя ослабить (утверждения 8-10). Предложен алгоритм, использующий указанную операцию, для построения комбинаторно полных матриц порядка 3 , у которых числа строк к числу столбцов сколь угодно мало (утверждение 11).

\section{1. Свойства столбцов комбинаторно полных матриц и их применение для оценки максимального числа столбцов}

В [4] доказана корректность (при $k \geqslant 2$ и $m \geqslant 2^{k}$ ) определения величины $n_{\max }^{(k)}(m)$, равной наибольшему числу $n$, при котором существует комбинаторно полная порядка $k$ матрица размера $m \times n$. Такая матрица называется максимальной комбинаторно полной порядка $k$ матрицей.

В [3] найдена точная формула для $n_{\max }^{(2)}(m)$ при $m \geqslant 4$ : 


$$
n_{\max }^{(2)}(m)= \begin{cases}\frac{1}{2} \cdot\left(\begin{array}{c}
2 r \\
r
\end{array}\right), & m=2 r, \\
\left(\begin{array}{c}
2 r \\
r-1
\end{array}\right), & m=2 r+1 .\end{cases}
$$

Для случая $k=3$ пока найдены только оценки величины $n_{\max }^{(3)}(m)$. Известно несколько точных значений $n_{\max }^{(3)}(m)$, в частности, в [1] установлено, что

$$
n_{\max }^{(3)}(8)=4, n_{\max }^{(3)}(9)=4, n_{\max }^{(3)}(10)=5 .
$$

Ниже приведены примеры максимальных комбинаторно полных матриц, соответствующих указанным значениям $n_{\max }^{(3)}(m)$ :

$$
\left(\begin{array}{l}
0000 \\
0011 \\
0101 \\
0110 \\
1001 \\
1010 \\
1100 \\
1111
\end{array}\right)_{8 \times 4},\left(\begin{array}{l}
0000 \\
0011 \\
0101 \\
0110 \\
1001 \\
1010 \\
1100 \\
1111 \\
0001
\end{array}\right)_{9 \times 4},\left(\begin{array}{l}
00001 \\
00111 \\
01011 \\
01100 \\
10011 \\
10100 \\
11000 \\
11110 \\
00010 \\
11101
\end{array}\right)_{10 \times 5}
$$

В конце раздела найдены точные значения $n_{\max }^{(3)}(11), n_{\max }^{(3)}(12), n_{\max }^{(3)}(13)$ и приведены комбинаторно полные матрицы порядка 3, на которых достигаются эти значения.

Докажем несколько утверждений о весах столбцов комбинаторно полных матриц.

Утверждение 1. Пусть $B$ - комбинаторно полная порядка $k$ матрица, имеющая $m$ строк. Тогда все столбци $B$ имеют вес не менее $2^{k-1}$ и не более $\left(m-2^{k-1}\right)$.

Доказательство. Пусть в матрице существует столбец веса менее $2^{k-1}$. Поскольку перестановка столбцов комбинаторно полной матрицы дает комбинаторно полную матрицу того же размера, то без ограничения общности считаем, что это первый столбец. Рассмотрим подматрицу $B$, содержащую этот столбец и любые другие $(k-1)$ столбцов. Обозначим ее $B^{\prime}$. Так как $B$ является комбинаторно полной порядка $k$, то матрица $B^{\prime}$ должна содержать все строки вида $(1, *, \ldots, *) \in V_{k}$. Таких строк $2^{k-1}$. Но так как вес первого столбца менее $2^{k-1}$, то $B^{\prime}$ содержит менее $2^{k-1}$ строк указанного вида, что приводит нас к противоречию.

Аналогичными рассуждениями можно показать, что если в матрице $B$ содержится столбец веса более $\left(m-2^{k-1}\right)$, то $B$ содержит подматрицу, в которой менее $2^{k-1}$ строк вида $(0, *, \ldots, *) \in V_{k}$, что противоречит определению комбинаторно полной матрицы.

Используя значение веса произвольного столбца комбинаторно полной матрицы, удается получить оценку общего числа столбцов.

Утверждение 2. Комбинаторно полная матрица порядка $k \geqslant 3$, содержащая столбеи, веса $m_{0}$, имеет не более $n_{\max }^{(k-1)}\left(m_{0}\right)+1$ столби,ов. 
Доказательство. Пусть комбинаторно полная матрица содержит столбец веса $m_{0}$. Перестановкой строк и столбцов получаем комбинаторно полную матрицу следующего вида:

$$
B=\left(\begin{array}{c|c}
0 & \\
\vdots & * \\
0 & \\
1 & \\
\vdots & C \\
1 &
\end{array}\right)_{m \times n}
$$

Так как $B$ - комбинаторно полная порядка $k$, то любая ее подматрица, состоящая из первого столбца и $k-1$ других произвольных столбцов, обладает следующим свойством. Для любого вектора $\left(\beta_{1}, \ldots, \beta_{k-1}\right) \in V_{k-1}$ в этой подматрице существует строка $\left(1, \beta_{1}, \ldots, \beta_{k-1}\right)$ среди нижних $m_{0}$ строк. Это равносильно тому, что матрица $C_{m_{0} \times(n-1)}$ является комбинаторно полной порядка $k-1$, а так как она содержит $m_{0}$ строк, то матрица $C$ имеет не более $n_{\max }^{(k-1)}\left(m_{0}\right)$ столбцов, поэтому $B$ имеет не более $n_{\max }^{(k-1)}\left(m_{0}\right)+1$ столбцов.

Отметим, что если инвертировать столбец веса $m_{0}$, то получим столбец веса $\left(m-m_{0}\right)$, где $m$ - число строк в рассматриваемой матрице. Поскольку инвертирование столбца комбинаторной полной матрицы дает комбинаторно полную матрицу того же порядка и размера, то получаем следствие утверждения 2.

Следствие 1. Комбинаторно полная матрища порядка $k$, содержащая $m$ строк и имеющая столбец веса $\left(m-m_{0}\right)$, всего содержит не более $n_{\max }^{(k-1)}\left(m_{0}\right)+1$ столбцов.

Важными частными случаями утверждения 2 являются экстремальные значения $m_{0} \in\left\{2^{k-1} ; m-2^{k-1}\right\}$.

Утверждение 3. Комбинаторно полная матрица порядка $k \geqslant 3$, содержащая $m$ строк и имеющая столбеи, веса $2^{k-1}$ или столбец, веса $\left(m-2^{k-1}\right)$, всего содержит не более $(k+1)$ столбиов.

Доказательство. Пусть матрица $B$ удовлетворяет условиям утверждения. По утверждению 2 , а также по следствию 1 , матрица $B$ имеет не более $n_{\max }^{(k-1)}\left(2^{k-1}\right)+1$ столбцов. Известно $([1])$, что $n_{\max }^{(k-1)}\left(2^{k-1}\right)=k$. Следовательно, $B$ имеет не более $(k+1)$ столбцов.

Опираясь на доказанное утверждение 2 и используя результаты работы [1], можно получить следующие оценки числа столбцов комбинаторно полной матрицы порядка $k$ для $k \geqslant 4$ (утверждение 4) и $k \geqslant 5$ (утверждение 5$)$.

Утверждение 4. Пусть $B$ - комбинаторно полная матрица порядка $k \geqslant 4$, содержащая $m$ строк и имеющая столбеи, веса не более $\left(2^{k-1}+k-2\right)$ или столбеи, веса не менее $m-\left(2^{k-1}+k-2\right)$. Тогда, если $k=4$, то B содержит не более 6 столбцов; если $k \geqslant 5$, то В содержит не более $(k+1)$ столбцов.

Доказательство. Пусть $B$ содержит столбец веса $m_{0}$, причем $m_{0} \leqslant\left(2^{k-1}+k-2\right)$. Тогда по утверждению 2 матрица $B$ имеет не более $n_{\max }^{(k-1)}\left(m_{0}\right)+1$ столбцов. В [1] доказано, что последовательность $n_{\max }^{(k)}(m)$ не убывает по $m$ при фиксированном значении $k$, то есть $n_{\max }^{(k)}(m) \leqslant n_{\max }^{(k)}(m+1)$. Следовательно, $n_{\max }^{(k-1)}\left(m_{0}\right) \leqslant$ 
$n_{\max }^{(k-1)}\left(2^{k-1}+k-2\right)$ и, значит, в матрице $B$ не более $n_{\max }^{(k-1)}\left(2^{k-1}+k-2\right)+1$ столбцов.

Пусть $k=4$. Подставляя в доказанное выше неравенство это значение, получим, что $n \leqslant n_{\max }^{(3)}(10)+1$. Как было сказано ранее, $n_{\max }^{(3)}(10)=5$. Следовательно, $n \leqslant 6$.

Пусть теперь $k \geqslant 5$. Известно ([1]), что в этом случае при $m=2^{k}+s$, $s<k$ справедливо равенство $n_{\max }^{(3)}(m)=k+1$. Следовательно, если $k \geqslant 5$, то $n_{\max }^{(k-1)}\left(2^{k-1}+k-2\right)=k$, поэтому $n \leqslant k+1$.

Для матриц, содержащих столбец веса не менее $m-\left(2^{k-1}+k-2\right)$, доказательство проводится аналогично с использованием следствия 1.

Утверждение 5. Пусть $B$ - комбинаторно полная порядка $k \geqslant 5$ матрица, содержащая $m$ строк и имеющая столбеи, веса не более $\left(2^{k-1}+2^{k-2}-1\right)$ или столбец веса не менее $m-\left(2^{k-1}+2^{k-2}-1\right)$. Тогда $B$ содержит не более $(k-1)$. $\left(2^{k-2}-k+2\right)+2$ столбцов.

Доказательство. Пусть матрица $B$ удовлетворяет условиям утверждения 5 . Аналогично доказательству предыдущего утверждения получаем, что матрица $B$ имеет не более $n_{\max }^{(k-1)}\left(2^{k-1}+2^{k-2}-1\right)+1$ столбцов. Известно [1], что при $k \geqslant 4$ и $k \leqslant m-2^{k}<2^{k-1}$ справедлива оценка $n_{\max }^{(k)}(m) \leqslant k\left(m-2^{k-1}-k+2\right)+1$. Используя эту оценку для значений $k-1 \geqslant 4$ (это выполнено по условию утверждения) и для значения $m^{\prime}=2^{k-1}+2^{k-2}-1$ (очевидно, что $k-1 \leqslant m^{\prime}-2^{k-1}=2^{k-2}-1<2^{k-2}$ ), получаем

$$
\begin{gathered}
n_{\max }^{(k-1)}\left(2^{k-1}+2^{k-2}-1\right)=n_{\max }^{(k-1)}\left(m^{\prime}\right) \leqslant(k-1) \cdot\left(m^{\prime}-2^{k-2}-(k-1)+2\right)+1= \\
=(k-1) \cdot\left(2^{k-1}-1-(k-1)+2\right)+1=(k-1) \cdot\left(2^{k-1}-k+2\right)+1 .
\end{gathered}
$$

Следовательно, $n_{\max }^{(k-1)}\left(2^{k-1}+2^{k-2}-1\right) \leqslant(k-1) \cdot\left(2^{k-1}-k+2\right)+1$, и $B$ содержит не более $(k-1) \cdot\left(2^{k-2}-k+2\right)+2$ столбцов.

Далее изучим случай $k=3$. Он интересен тем, что при малых величинах $m$ можно использовать компьютерный перебор для нахождения значений функции $n_{\max }^{(3)}(m)$. Как указано выше, уже найдены точные значения $n_{\max }^{(3)}(m)$ при $m \in\{8,9,10\}$. Опираясь на утверждения $1-3$, а также используя некоторые матрицы, найденные при помощи вычислительной техники, далее получены точные значения также для $m \in\{11,12,13\}$.

Утверждение 6. Вернъ равенства

$$
n_{\max }^{(3)}(11)=5, \quad n_{\max }^{(3)}(12)=11, \quad n_{\max }^{(3)}(13)=11 .
$$

Доказательство. Пусть $B$ - максимальная комбинаторно полная порядка 3 матрица размера $m \times n$. Напомним, что максимальной порядка $k$ называется комбинаторно полная матрица размера $m \times n_{\max }^{(k)}(m)$.

Пусть $m=11$. Воспользуемся тем, что функция $n_{\max }^{(k)}(m)$ при фиксированном $k$ является не убывающей по $m$. Следовательно, $n_{\max }^{(3)}(11) \geqslant n_{\max }^{(3)}(10)=5$. По утверждению 1 столбцы $B$ могут быть только веса не менее 4 и не более 7 , причем если $B$ содержит столбец веса 4 или 7 , то $n \leqslant 4$ по утверждению 3 , и матрица $B$ в этом случае не является максимальной. Итак, все столбцы матрицы $B$ имеют вес 5 или 6. Тогда по утверждению 2 и следствию 1 матрица $B$ содержит не более 
$n_{\max }^{(2)}(5)+1$ столбцов. Используя формулу для вычисления $n_{\max }^{(2)}(m)$, найденную в [3] и приведенную в начале раздела, получаем $n_{\max }^{(2)}(5)=4$, поэтому $n_{\max }^{(3)}(11) \leqslant 5$. Покажем, что эта оценка достижима. Рассмотрим следующую матрицу:

$$
C=\left(\begin{array}{l}
11101000010 \\
01101001100 \\
01010101010 \\
00110011010 \\
00001111010
\end{array}\right)_{5 \times 11}
$$

Непосредственной проверкой можно убедиться в том, что матрица $C^{T}$ является комбинаторно полной порядка 3 . Следовательно, $n_{\max }^{(3)}(11)=5$.

Пусть $m=12$. Из монотонности по $m$ функции $n_{\max }^{(k)}(m)$ при фиксированном $k$ следует, что $n_{\max }^{(3)}(12) \geqslant n_{\max }^{(3)}(11)=5$. По утверждению 1 столбцы $B$ могут иметь значение веса от 4 до 8 . Если $B$ содержит столбец веса 4 или 8 , то $n \leqslant 4$ по утверждению 3 , и матрица $B$ в этом случае не является максимальной. Значит, вес столбцов $B$ может принимать значения 5,6 или 7. Если $B$ содержит столбец веса 5 или 7 , то по утверждению 2 и следствию 1 матрица $B$ содержит не более $n_{\max }^{(2)}(5)+1$ столбцов, то есть $n \leqslant 5$. Если $B$ состоит только из столбцов веса 6 , то $B$ содержит не более $n_{\max }^{(2)}(6)+1$ столбцов. Используя формулу для $n_{\max }^{(2)}(m)$, указанную в начале раздела, получаем $n_{\max }^{(2)}(6)=10$, поэтому $n_{\max }^{(3)}(12) \leqslant 11$. Приведем пример матрицы, для которой достигается эта оценка:

$$
A=\left(\begin{array}{l}
00000000000 \\
00100011111 \\
01001100111 \\
01110101001 \\
10010110011 \\
10101101010 \\
11001011001 \\
11100110100 \\
00011111100 \\
01111010010 \\
10111000101 \\
11010001110
\end{array}\right)_{12 \times 11}
$$

Непосредственной проверкой можно убедиться, что матрица $A$ является комбинаторно полной порядка 3 . Следовательно, $n_{\max }^{(3)}(12)=11$.

Пусть $m=13$. Согласно утверждению 1 столбцы $B$ могут быть только веса не менее 4 и не более 9 . Если $B$ содержит столбец веса 4 или 9 , то по утверждению 3 верно неравенство $n \leqslant 4$, и матрица $B$ в этом случае не является максимальной. Если $B$ содержит столбец веса 5 или 8 , то матрица $B$ содержит не более $n_{\max }^{(2)}(5)+1$ столбцов, то есть $n \leqslant 5$, и матрица $B$ в этом случае также не является максимальной. Итак, $B$ может иметь только столбцы веса 6 или 7. В обоих случаях по утверждению 2 и следствию 1 матрица $B$ содержит не более $n_{\max }^{(2)}(6)+1$ столбцов, то есть $n \leqslant 11$. Следовательно, $n_{\max }^{(3)}(13) \leqslant 11$. С другой стороны, из монотонности по $m$ функции $n_{\max }^{(k)}(m)$ при фиксированном $k$ следует, что $n_{\max }^{(3)}(13) \geqslant n_{\max }^{(3)}(12)=11$. Таким образом, $n_{\max }^{(3)}(13)=11$. Чтобы предъявить комбинаторно полную порядка 
3 матрицу, содержащую 13 строк и 11 столбцов, достаточно добавить любую новую строку к матрице $A$, приведенной выше.

Отметим, что приведенные в доказательстве утверждения 6 матрицы $A$ и $C$ найдены экспериментально. При числе строк $m \geqslant 14$ задача нахождения максимальной комбинаторно полной матрицы порядка 3 имеет высокую трудоемкость, и именно это является препятствием к вычислению остальных значений функции $n_{\max }^{(3)}(m)$.

\section{2. Введение комбинаторно достаточных матриц и их свойства}

В предыдущем разделе было показано, что при $m \leqslant 13$ для всех комбинаторно полных порядка 3 матриц размера $m \times n$ справедливо соотношение $m>n$. С другой стороны, доказано ([5]), что существует комбинаторно полная матрица размера $m \times n$ порядка $k \geqslant 3$, где числа $m, n$ и $k$ удовлетворяют неравенству $m \leqslant 2^{k} \cdot k \cdot \ln n$. Для таких матриц отношение $\frac{m}{n}$ с ростом $n$ становится сколь угодно малым. Однако предложенные ранее алгоритмы построения комбинаторно полных матриц ([2], [5]) либо дают матрицы, для которых $m$ значительно больше $n$, либо имеют слишком высокую трудоемкость. Например, сложность существующих алгоритмов построения комбинаторно полной матрицы порядка 3 , для которой $m \leqslant n$, лежит за границами практически достижимых величин. Поэтому далее в разделе 3 предложен новый алгоритм построения комбинаторно полных матриц порядка 3. Этот алгоритм основан на понятии комбинаторно достаточной матрицы и бинарной операции на множестве двоичных матриц, которые определены ниже.

Введем следующее определение. Двоичную матрицу $T$ размера $m \times n, n \geqslant k$, будем называть комбинаторно достаточной порядка $k$, если матрица $\left(\begin{array}{c}\bar{T} \\ T\end{array}\right)$ является комбинаторно полной порядка $k$ (здесь $\bar{T}$ - матрица, полученная из $T$ инвертированием всех ее элементов).

В терминах булевых функций понятие комбинаторно достаточной матрицы имеет следующий вид. Для булевой функции $f\left(x_{1}, \ldots, x_{n}\right)$ матрица $E_{f}$ (строками которой являются все векторы множества $\left.E_{f}\right)$ является комбинаторно достаточной порядка $k$ тогда и только тогда, когда матрица $\left(\begin{array}{c}\overline{E_{f}} \\ E_{f}\end{array}\right)$ является комбинаторно полной порядка $k$; последнее, в свою очередь, эквивалентно тому, что функция $f\left(x_{1}, \ldots, x_{n}\right) \bigvee f\left(\overline{x_{1}}, \ldots, \overline{x_{n}}\right)$ не имеет имплицент от $k$ переменных.

Нетрудно указать связь максимального числа столбцов комбинаторно достаточных матриц с параметром $n_{\max }^{(k)}(m)$.

Утверждение 7. Пусть $B$ - комбинаторно достаточная порядка $k$ матрица, содержащая $m$ строк. Тогда В имеет не более $n_{\max }^{(k)}(2 m)$ столбцов.

Очевидно, любая комбинаторно полная матрица является комбинаторно достаточной того же порядка. При $k=3$ простым примером комбинаторно достаточной матрицы является единичная матрица размера $n \times n, n \geqslant 4$. Действительно, любые 3 столбца единичной матрицы содержат строки (000), (100), (010), (001). Данные строки вместе со своими отрицаниями образуют множество $V_{3}$. Приведем некоторые простые свойства комбинаторно достаточных матриц. Пусть $B$ - комбинаторно достаточная порядка $k$ матрица размера $m \times n, n \geqslant k$. Тогда: 
1) $m \geqslant 2^{k-1}$

2) если $k>1$, то $B$ является комбинаторно достаточной порядка $k^{\prime}$ для любого $k^{\prime} \in\{1, \ldots, k-1\}$

3) любая подматрица $B$, содержащая $m$ строк и не менее $k$ столбцов, является комбинаторно достаточной порядка $k$;

4) если матрица $B^{\prime}$ получена из матрицы $B$ инвертированием любых строк и столбиов, то $B^{\prime}$ является комбинаторно достаточной порядка $k$;

5) если матрица $B^{\prime}$ получена из матрицы $B$ произволъными перестановками ее строк и столбцов, то $B^{\prime}$ является комбинаторно достаточной порядка $k$;

6) если матрица $B^{\prime}$ получена из матрицы $B$ добавлением любого числа произволъных строк, то $B^{\prime}$ является комбинаторно достаточной порядка $k$.

Отметим, что в отличие от комбинаторно полных матриц, комбинаторно достаточные могут содержать любые столбцы. Действительно, инвертированием некоторых строк можно добиться наличия в каждой двоичной матрице любого произвольного столбца.

Одним из ожидаемых способов построения комбинаторно полных матриц является попытка использовать для этого тензорное умножение матриц. Однако, вообще говоря, тензорное произведение комбинаторно полных матриц - не комбинаторно полная матрица. Ниже доказано, что если $A$ и $T$ являются комбинаторно полными матрицами порядка 3 , то матрица ${ }^{1} T \otimes A \oplus \bar{T} \otimes \bar{A}$ (здесь « $\otimes »-$ тензорное произведение матриц, «Ф» - поэлементное сложение матриц по модулю 2) является комбинаторно полной. Основным результатом этого раздела является утверждение о том, что какая-либо одна из матриц $A$ и $T$ не обязательно должна быть комбинаторно полной, - она может быть комбинаторно достаточной. Свойство комбинаторной достаточности является менее сильным, чем свойство комбинаторной полноты, поэтому справедливо ожидать, что при фиксированных $m$ и $k$ комбинаторно достаточная порядка $k$ матрица, содержащая $m$ строк, может иметь более $n_{\max }^{(k)}(m)$ столбцов. Поэтому при построении новой матрицы из матриц $A$ и $T$ по указанной формуле удастся добиться уменьшения отношения числа строк по отношению к числу столбцов. Этот результат доказан в разделе 3 .

Далее для любого $\varepsilon \in\{0,1\}$ обозначим $A^{\varepsilon}=\left\{\begin{array}{l}A, \varepsilon=1 \\ \bar{A}, \varepsilon=0\end{array}\right.$. Пусть $T=\left(t_{i, j}\right)_{r \times s}$. Тогда

$$
B=T \otimes A \oplus \bar{T} \otimes \bar{A}=\left(\begin{array}{ccc}
A^{t_{1,1}} & \cdots & A^{t_{1, s}} \\
\vdots & \ddots & \vdots \\
A^{t_{r, 1}} & \cdots & A^{t_{r, s}}
\end{array}\right) .
$$

Для любого $i \in\{1, \ldots, r\}$ будем называть $i$-й блок-строкой матрицы $B$ ее подматрицу размера $m \times(s \cdot n)$ следующего вида: $\vec{B}^{[i]}=\left(A^{t_{i, 1}} A^{t_{i, 2}} \ldots A^{t_{i, s}}\right)$; а $j$-м блок-столбцом $(j \in\{1, \ldots, s\})$ назовем подматрицу $B$ размера $(r \cdot m) \times n$ следующего вида: $B^{\downarrow[j]}=\left(\begin{array}{lll}A^{t_{1, j}} & A^{t_{2, j}} \ldots A^{t_{r, j}}\end{array}\right)^{T}$.

Теорема 1. Пусть матрица А размера $m \times n$ является комбинаторно полной порядка 3, и пусть матрица $T$ размера $r \times s$ является комбинаторно достаточной порядка 3. Тогда матрица $B=T \otimes A \oplus \bar{T} \otimes \bar{A}$ размера $(r \cdot m) \times(s \cdot n)$ является комбинаторно полной порядка 3.

1 Чтобы не загромождать формулы скобками, примем следующий порядок: сначала выполняются все операции тензорного произведения, затем суммирование матриц. 
Доказательство. Для доказательства комбинаторной полноты порядка 3 матрицы $B$ возьмем 3 любых ее столбца $B_{i_{1}}^{\downarrow}, B_{i_{2}}^{\downarrow}, B_{i_{3}}^{\downarrow}$, причем $i_{l}=\left(p_{l}-1\right) n+n_{l}$ для любого $l \in\{1,2,3\}$, где $p_{l} \in \overline{1, s}$ - номер блок-столбца, в котором находится $B_{i_{l}}^{\downarrow}$, а $n_{l} \in\{1, \ldots, n\}$ - номер столбца $B_{i_{l}}^{\downarrow}$ в блок-столбце $B^{\downarrow\left[p_{l}\right]}$ (рис. 1). Рассмотрим отдельно три случая, которые исчерпывают все варианты выбора столбцов $B_{i_{1}}^{\downarrow}, B_{i_{2}}^{\downarrow}, B_{i_{3}}^{\downarrow}$. Обозначим $B^{\prime}=\left(\begin{array}{lll}B_{i_{1}}^{\downarrow} & B_{i_{2}}^{\downarrow} & B_{i_{3}}^{\downarrow}\end{array}\right), T^{\prime}=\left(T_{p_{1}}^{\downarrow} T_{p_{2}}^{\downarrow} T_{p_{3}}^{\downarrow}\right)$.

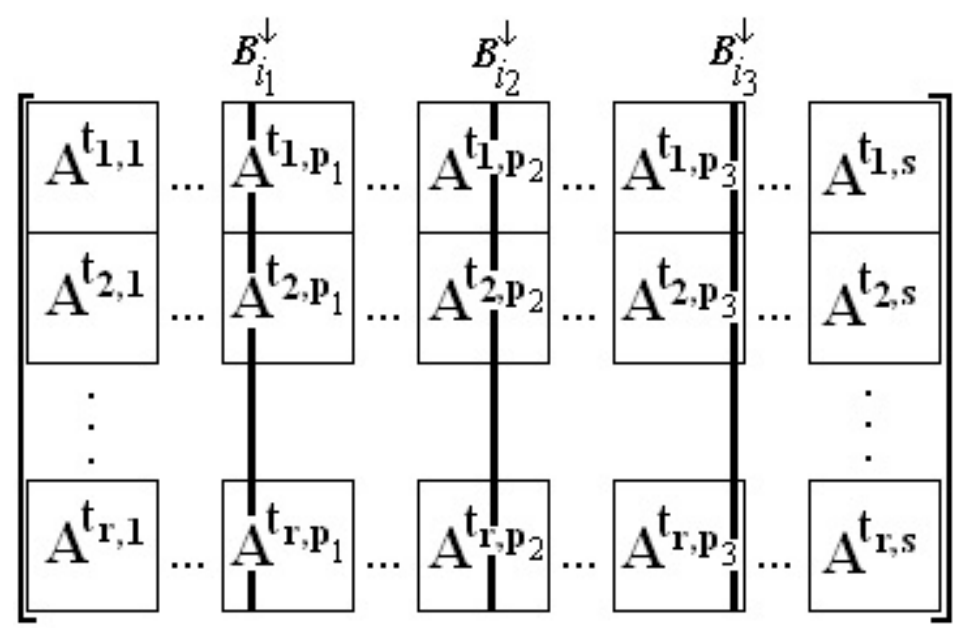

Рис. 1. Первый вариант выбора столбцов.

Первый случай: $p_{1}, p_{2}, p_{3}$ попарно не равны, то есть выбранные столбцы матрицы $B$ принадлежат трем различным блок-столбцам (рис. 1).

Пусть $n_{1}, n_{2}, n_{3}$ попарно не равны. Так как матрица $T$ является комбинаторно достаточной порядка 3 , то в матрице $T^{\prime}$ существует строка (111) или (000). Пусть это строка $\vec{T}_{l_{1}}$. Рассмотрим подматрицу $B$, образованную пересечением блок-строки $\vec{B}^{\left[l_{1}\right]}$ и матрицы $\left(B^{\downarrow\left[p_{1}\right]} B^{\downarrow\left[p_{2}\right]} B^{\downarrow\left[p_{3}\right]}\right)$. Тогда эта подматрица будет иметь вид: $(A A A)$ или $(\bar{A} \bar{A} \bar{A})$. Следовательно, матрица $B^{\prime}$ содержит подматрицу следующего вида: $\left(A_{n_{1}}^{\downarrow} A_{n_{2}}^{\downarrow} A_{n_{3}}^{\downarrow}\right)$ или $\left(\bar{A}_{n_{1}}^{\downarrow} \bar{A}_{n_{2}}^{\downarrow} \bar{A}_{n_{3}}^{\downarrow}\right)$. Ввиду комбинаторной полноты матриц $A$ и $\bar{A}$ матрица $B^{\prime}$ содержит все строки длины 3 .

Пусть ровно два элемента из $n_{1}, n_{2}, n_{3}$ равны. Положим без ограничения общности $n_{1}=n_{2} \neq n_{3}$. Снова рассмотрим подматрицу, образованную пересечением блок-строки $\vec{B}^{\left[l_{1}\right]}$ и матрицы $\left(B^{\downarrow\left[p_{1}\right]} B^{\downarrow\left[p_{2}\right]} B^{\downarrow\left[p_{3}\right]}\right)$, имеющую вид $(A A A)$ или $(\bar{A} \bar{A} \bar{A})$. Следовательно, матрица $B^{\prime}$ содержит подматрицу вида $\left(A_{n_{1}}^{\downarrow} A_{n_{1}}^{\downarrow} A_{n_{3}}^{\downarrow}\right)$ или $\left(\bar{A}_{n_{1}}^{\downarrow} \bar{A}_{n_{1}}^{\downarrow} \bar{A}_{n_{3}}^{\downarrow}\right)$. Нетрудно видеть, что ввиду комбинаторной полноты матриц $A$ и $\bar{A}$ в этом случае $B^{\prime}$ содержит следующие строки: (000), (001), (110), (111). Далее, так как матрица $T$ является комбинаторно достаточной порядка 3 , то в матрице $T^{\prime}$ существует строка (100) или $(011)$. Пусть это строка $\overrightarrow{T^{\prime}} l_{2}$. Тогда рассмотрим подматрицу $B$, образованную пересечением матрицы $\left(B^{\downarrow\left[p_{1}\right]} B^{\downarrow\left[p_{2}\right]} B^{\downarrow\left[p_{3}\right]}\right)$ и блок-строки $\vec{B}^{\left[l_{2}\right]}$. Эта подматрица имеет вид $(\bar{A} A A)$ или $(A \bar{A} \bar{A})$. Следовательно, матрица $B^{\prime}$ содержит подматрицу вида $\left(\bar{A}_{n_{1}}^{\downarrow} A_{n_{1}}^{\downarrow} A_{n_{3}}^{\downarrow}\right)$ или $\left(A_{n_{1}}^{\downarrow} \bar{A}_{n_{1}}^{\downarrow} \bar{A}_{n_{3}}^{\downarrow}\right)$. Нетрудно видеть, 
что ввиду комбинаторной полноты матриц $A$ и $\bar{A}$ в этом случае в $B^{\prime}$ также содержатся следующие строки: (100), (101), (010), (011). Следовательно, $B^{\prime}$ содержит все строки длины 3 .

Пусть $n_{1}=n_{2}=n_{3}$. Покажем, что для любого $\vec{\alpha} \in V_{3}$ существует строка в $B^{\prime}$, равная $\vec{\alpha}$. Пусть $\vec{\alpha}=\left(\alpha_{1}, \alpha_{2}, \alpha_{3}\right)$. Так как матрица $T-$ комбинаторно достаточная порядка 3 , то в матрице $T^{\prime}$ содержится строка $\vec{\alpha}$ или ее отрицание. Пусть это строка $\vec{T}_{l_{3}}^{\prime}$. Рассмотрим подматрицу, образованную пересечением матрицы $\left(B^{\downarrow\left[p_{1}\right]} B^{\downarrow\left[p_{2}\right]} B^{\downarrow\left[p_{3}\right]}\right)$ и блок-строки $\vec{B}^{\left[l_{3}\right]}$. Эта подматрица имеет вид $\left(A^{\alpha_{1}} A^{\alpha_{2}} A^{\alpha_{3}}\right)$ или $\left(\bar{A}^{\alpha_{1}} \bar{A}^{\alpha_{2}} \bar{A}^{\alpha_{3}}\right)$. Следовательно, матрица $B^{\prime}$ содержит подматрицу следующего вида:

$$
\left(\left(A_{n_{1}}^{\downarrow}\right)^{\alpha_{1}}\left(A_{n_{1}}^{\downarrow}\right)^{\alpha_{2}}\left(A_{n_{1}}^{\downarrow}\right)^{\alpha_{3}}\right) \text { или }\left(\left(\bar{A}_{n_{1}}^{\downarrow}\right)^{\alpha_{1}}\left(\bar{A}_{n_{1}}^{\downarrow}\right)^{\alpha_{2}}\left(\bar{A}_{n_{1}}^{\downarrow}\right)^{\alpha_{3}}\right) .
$$

Из комбинаторной полноты матриц $A$ и $\bar{A}$ следует, что обе приведенные подматрицы содержат строку $\left(1^{\alpha_{1}} 1^{\alpha_{2}} 1^{\alpha_{3}}\right)$, поэтому $B^{\prime}$ содержит строку $\vec{\alpha}$.

Второй случай: ровно два элемента из $p_{1}, p_{2}, p_{3}$ равны, то есть выбранные столбцы $B_{i_{1}}^{\downarrow}, B_{i_{2}}^{\downarrow}, B_{i_{3}}^{\downarrow}$ лежат в двух блок-столбцах. Положим без ограничения общности $p_{1}=$ $p_{2} \neq p_{3}$. Следовательно, $n_{1} \neq n_{2}$ (рис. 2). Обозначим $T^{\prime \prime}=\left(T_{p_{1}}^{\downarrow} T_{p_{3}}^{\downarrow}\right)$.

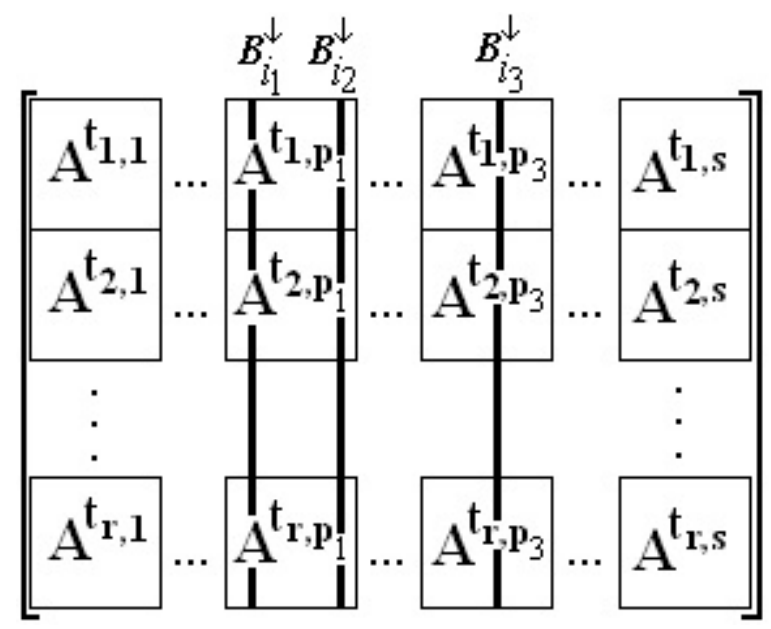

Рис. 2. Второй вариант выбора столбцов.

Пусть $n_{3} \notin\left\{n_{1}, n_{2}\right\}$. Нетрудно видеть, что так как матрица $T$ является комбинаторно достаточной порядка 3 , то в матрице $T^{\prime \prime}$ существует строка, равная (00) или (11). Пусть это строка $\overrightarrow{T^{\prime \prime}} l_{4}$. Рассмотрим подматрицу $B$, образованную пересечением матрицы $\left(B^{\downarrow\left[p_{1}\right]} B^{\downarrow\left[p_{3}\right]}\right)$ и блок-строки $\vec{B}^{\left[l_{4}\right]}$. Эта подматрица имеет вид $(A A)$ или $(\bar{A} \bar{A})$. Следовательно, матрица $B^{\prime}$ содержит подматрицу вида $\left(A_{n_{1}}^{\downarrow} A_{n_{2}}^{\downarrow} A_{n_{3}}^{\downarrow}\right)$ или $\left(\bar{A}_{n_{1}}^{\downarrow} \bar{A}_{n_{2}}^{\downarrow} \bar{A}_{n_{3}}^{\downarrow}\right)$. Ввиду комбинаторной полноты матриц $A$ и $\bar{A}$ матрица $B^{\prime}$ содержит все строки длины 3.

Пусть $n_{3} \in\left\{n_{1}, n_{2}\right\}$. Положим без ограничения общности $n_{2}=n_{3}$. Нетрудно убедиться, что так как $T$ является комбинаторно достаточной порядка 3 , то $T^{\prime \prime}$ содержит одну из следующих пар строк: $\{(01),(00)\},\{(10),(00)\},\{(01),(11)\},\{(10),(11)\}$. 
Пусть это строки $\vec{T}_{l_{5}}$ и $\overrightarrow{T^{\prime \prime}} l_{6}$. Тогда рассмотрим подматрицу $B$, образованную пересечением матрицы $\left(B^{\downarrow\left[p_{2}\right]} B^{\downarrow\left[p_{3}\right]}\right)$ и матрицы $\left(\begin{array}{c}\vec{B}^{\left[l_{5}\right]} \\ \vec{B}^{\left[l_{6}\right]}\end{array}\right)$. Эта подматрица имеет один из следующих видов: $\left(\begin{array}{c}\bar{A} A \\ \bar{A} \\ \bar{A}\end{array}\right),\left(\begin{array}{c}A \bar{A} \\ \bar{A} \bar{A}\end{array}\right),\left(\begin{array}{c}\bar{A} A \\ A\end{array}\right)$ или $\left(\begin{array}{c}A \bar{A} \\ A\end{array}\right)$ (с точностью до перестановки блок-строк). Следовательно, матрица $B^{\prime}$ содержит одну из подматриц

$$
\left(\begin{array}{ccc}
\bar{A}_{n_{1}}^{\downarrow} & \bar{A}_{n_{2}}^{\downarrow} & A_{n_{2}}^{\downarrow} \\
\bar{A}_{n_{1}}^{\downarrow} & \bar{A}_{n_{2}}^{\downarrow} & \bar{A}_{n_{2}}^{\downarrow}
\end{array}\right),\left(\begin{array}{ccc}
A_{n_{1}}^{\downarrow} & A_{n_{2}}^{\downarrow} & \bar{A}_{n_{2}}^{\downarrow} \\
\bar{A}_{n_{1}}^{\downarrow} & \bar{A}_{n_{2}}^{\downarrow} & \bar{A}_{n_{2}}^{\downarrow}
\end{array}\right),\left(\begin{array}{ccc}
\bar{A}_{n_{1}}^{\downarrow} & \bar{A}_{n_{2}}^{\downarrow} & A_{n_{2}}^{\downarrow} \\
A_{n_{1}}^{\downarrow} & A_{n_{2}}^{\downarrow} & A_{n_{2}}^{\downarrow}
\end{array}\right),\left(\begin{array}{ccc}
A_{n_{1}}^{\downarrow} & A_{n_{2}}^{\downarrow} & \bar{A}_{n_{2}}^{\downarrow} \\
A_{n_{1}}^{\downarrow} & A_{n_{2}}^{\downarrow} & A_{n_{2}}^{\downarrow}
\end{array}\right) .
$$

Из комбинаторной полноты матриц $A$ и $\bar{A}$ легко следует, что во всех четырех случаях $B^{\prime}$ содержит все возможные строки длины 3.

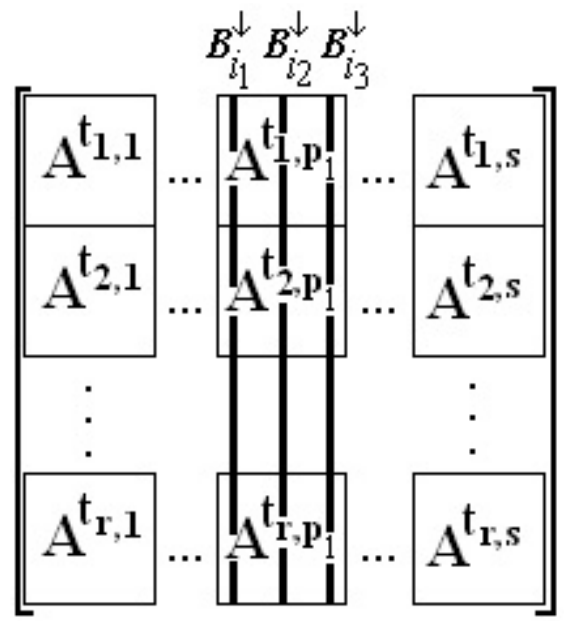

Рис. 3. Третий вариант выбора столбцов.

Третий случай: $p_{1}=p_{2}=p_{3}$, то есть выбранные столбцы $B_{i_{1}}^{\downarrow}, B_{i_{2}}^{\downarrow}, B_{i_{3}}^{\downarrow}$ лежат в одном блок-столбце $B^{\downarrow\left[p_{1}\right]}$ (рис. 3). Этот блок-столбец содержит $A$ или $\bar{A}$ как подматрицу, поэтому матрица $B^{\prime}$ содержит все возможные строки длины 3.

Покажем, что матрицы $A$ и $T$ можно менять местами.

Следствие 2. В условиях теоремы 1 матрица $A \otimes T \oplus \bar{A} \otimes \bar{T}$ является комбинаторно полной порядка 3.

Доказательство. Известно ([7]), что для тензорного произведения матриц $A \otimes T$ существуют такие подстановочные матрицы $P$ и $Q$, что $P \cdot(A \otimes T) \cdot Q=T \otimes A$. Тогда, очевидно, $P \cdot(\bar{A} \otimes \bar{T}) \cdot Q=\bar{T} \otimes \bar{A}$ и $P \cdot(A \otimes T \oplus \bar{A} \otimes \bar{T}) \cdot Q=P \cdot(A \otimes T) \cdot Q \oplus P$. $(\bar{A} \otimes \bar{T}) \cdot Q=T \otimes A \oplus \bar{T} \otimes \bar{A}$. По теореме 1 матрица $T \otimes A \oplus \bar{T} \otimes \bar{A}$ комбинаторно полная порядка 3. Поскольку при перестановке строк или столбцов комбинаторно полной матрицы получается комбинаторно полная матрица того же порядка, то матрица $A \otimes T \oplus \bar{A} \otimes \bar{T}=P^{-1} \cdot(T \otimes A \oplus \bar{T} \otimes \bar{A}) \cdot Q^{-1}$ комбинаторно полная порядка 3. 
Далее утверждениями 8, 9 и примерами показывается, что в теореме 1 нельзя ослабить условия комбинаторной полноты матрицы $A$ и комбинаторной достаточности матрицы $T$. Итоговые результаты сведены в таблицу 1 , приведенную ниже.

Утверждение 8. Для любой двоичной матрицъ А, не являющейся комбинаторно полной порядка 3 , существует такая комбинаторно достаточная матрица $T$ порядка 3 , что матрица $B=T \otimes A \oplus \bar{T} \otimes \bar{A}$ не является комбинаторно полной порядка 3.

Доказательство. Так как матрица $A$ не является комбинаторно полной порядка 3 , то существуют двоичный вектор $\left(\alpha_{1}, \alpha_{2}, \alpha_{3}\right) \in V_{3}$ и подматрица $A$, состоящая из трех столбцов, которая не содержит строку $\left(\alpha_{1}, \alpha_{2}, \alpha_{3}\right)$. Без ограничения общности будем считать, что это первые три столбца. Рассмотрим матрицу

$$
T=\left(\begin{array}{l}
100 \\
101 \\
110 \\
111
\end{array}\right)
$$

Очевидно, $T$ является комбинаторно достаточной порядка 3 . Тогда матрица $B=$ $T \otimes A \oplus \bar{T} \otimes \bar{A}$ имеет следующий вид:

$$
B=\left(\begin{array}{c}
A \overline{A A} \\
A \bar{A} A \\
A A \bar{A} \\
A A A
\end{array}\right) .
$$

Нетрудно видеть, что первые 3 столбца матрицы $B$ не содержат строку $\left(\alpha_{1}, \alpha_{2}, \alpha_{3}\right)$.

Дополним утверждение 8: две комбинаторно достаточные матрицы могут дать при «перемножении» по указанной формуле комбинаторно полную матрицу порядка 3. Приведем пример.

Пример 1. Рассмотрим следующие двоичные матрицы $A$ и $T$ :

$$
A=\left(\begin{array}{l}
001 \\
010 \\
011 \\
100 \\
101 \\
110 \\
111
\end{array}\right), T=\left(\begin{array}{l}
1000 \\
0100 \\
0010 \\
0001 \\
1111
\end{array}\right)
$$

Нетрудно видеть, что обе матрицы $A$ и $T$ являются комбинаторно достаточными порядка 3 , но не являются комбинаторно полными порядка 3. Рассмотрим матрицу $B=T \otimes A \oplus \bar{T} \otimes \bar{A}$ (для компактности изложения приведем матрицу $B^{T}$, транспонированную к $B$ ): 


$$
B^{T}=\left(\begin{array}{c}
01010100101010010101010101011010101 \\
10011001001100100110001100110110011 \\
11100001110000111000000011110001111 \\
01010100101010101010101010101010101 \\
10011001001100011001110011000110011 \\
11100001110000000111111100000001111 \\
01010101010101010101001010101010101 \\
10011000110011100110010011000110011 \\
11100000001111111000011100000001111 \\
10101010101010010101001010101010101 \\
01100111001100100110010011000110011 \\
00011111110000111000011100000001111
\end{array}\right) .
$$

Матрица $B$ является комбинаторно полной порядка 3. В этом можно убедиться непосредственной проверкой или проведя рассуждения, аналогичные использованным в доказательстве теоремы 1.

Докажем, что матрицы $A$ и $T$ обязаны быть комбинаторно достаточными порядка 3.

Утверждение 9. Если матрица А имеет не менее трех столбцов и не является комбинаторно достаточной порядка 3 , то для любой двоичной матрицы Т матрица $B=T \otimes A \oplus \bar{T} \otimes \bar{A}$ не является комбинаторно полной порядка 3 . Если матрица T имеет не менее трех столбцов и не является комбинаторно достаточной порядка 3, то для любой двоичной матрицы $A$ матрица $B=T \otimes A \oplus \bar{T} \otimes \bar{A}$ также не является комбинаторно полной порядка 3.

Доказательство. Пусть матрица $A$, содержащая хотя бы 3 столбца, не является комбинаторно достаточной порядка 3 . Тогда в любом блок-столбце матрицы $B=$ $T \otimes A \oplus \bar{T} \otimes \bar{A}$ будут только строки, содержащиеся в $A$ или в $\bar{A}$. Поскольку $A$ не является комбинаторно достаточной порядка 3 , любой блок-столбец матрицы $B$ не является комбинаторно полной порядка 3 матрицей. Следовательно, матрица $B$ не является комбинаторно полной порядка 3.

Пусть теперь матрица $T$ имеет не менее трех столбцов и не является комбинаторно достаточной порядка 3 . Предположим, что найдется такая матрица $A$, что $B=T \otimes A \oplus \bar{T} \otimes \bar{A}$ - комбинаторно полная порядка 3. Тогда, как и при доказательстве следствия 2 , воспользуемся тем, что существуют такие подстановочные матрицы $P$ и $Q$, что $P \cdot(T \otimes A) \cdot Q=A \otimes T$. Следовательно, $P \cdot(T \otimes A \oplus \bar{T} \otimes \bar{A}) \cdot Q=A \otimes T \oplus \bar{A} \otimes \bar{T}$. Поскольку при перестановке строк и столбцов комбинаторно полной матрицы получается комбинаторно полная матрица того же порядка, то матрица $A \otimes T \oplus \bar{A} \otimes \bar{T}$ является комбинаторно полной порядка 3. Однако, согласно доказанному выше, если матрица $T$ не является комбинаторно достаточной порядка 3 , то матрица $A \otimes T \oplus \bar{A} \otimes \bar{T}$ не является комбинаторно полной порядка 3 для любой двоичной матрицы $A$ - противоречие.

В примере 1 с помощью двух комбинаторно достаточных, но не комбинаторно полных порядка 3 матриц $A$ и $T$ образована комбинаторно полная порядка 3 матрица $B$. Такой результат получается не для любой пары комбинаторно достаточных матриц.

Пример 2. Рассмотрим следующие двоичные матрицы $A$ и $T$ : 


$$
A=T=\left(\begin{array}{l}
1000 \\
0100 \\
0010 \\
0001
\end{array}\right)
$$

Нетрудно видеть, что матрицы $A$ и $T$ являются комбинаторно достаточными, но не комбинаторно полными порядка 3 . Тогда матрица $B=T \otimes A \bigoplus \bar{T} \otimes \bar{A}$ имеет вид

$$
B=\left(\begin{array}{l}
1000011101110111 \\
0100101110111011 \\
0010110111011101 \\
0001111011101110 \\
0111100001110111 \\
1011010010111011 \\
1101001011011101 \\
1110000111101110 \\
0111011110000111 \\
1011101101001011 \\
1101110100101101 \\
1110111000011110 \\
0111011101111000 \\
1011101110110100 \\
1101110111010010 \\
1110111011100001
\end{array}\right) .
$$

В её подматрице, составленной из столбцов $B_{1}^{\downarrow}, B_{2}^{\downarrow}, B_{5}^{\downarrow}$, отсутствует строка (000). Следовательно, матрица $B$ не является комбинаторно полной порядка 3.

Таким образом, теорема 1 , следствие 2 , утверждения 8,9 и примеры 1,2 позволяют проиллюстрировать следующей таблицей свойства матрицы $B=T \otimes A \oplus \bar{T} \otimes \bar{A}$ в зависимости от свойств двоичных матриц $A$ и $T$.

\begin{tabular}{|l|l|l|l|}
\hline \multicolumn{1}{|c|}{$\backslash A$} & к.п. & к.Д. & н.к.д. \\
\hline к.п. & + & + & - \\
\hline к.д. & + & \pm & - \\
\hline н.к.д. & - & - & - \\
\hline
\end{tabular}

Здесь «к.п.»- комбинаторно полная порядка 3 ; «к.д.»- комбинаторно достаточная порядка 3 ; «н.к.д.» - не является комбинаторно достаточной порядка 3 ; «十» - матрица $B$ является комбинаторно полной порядка 3 ; «土»- матрица $B$ может как являться, так и не являться комбинаторно полной порядка 3 в зависимости от конкретных матриц $A$ и $T$; «-»- матрица $B$ не является комбинаторно полной порядка 3.

Таким образом, гарантировать комбинаторную полноту порядка 3 матрицы $B$ можно только в случае, когда одна из матриц $A$ или $T$ является комбинаторно полной, а другая - комбинаторно достаточной порядка 3.

Отметим, что мы не случайно ограничиваемся случаем комбинаторной достаточности порядка 3. Оказывается, что для $k \geqslant 4$ при соблюдении аналогичных условий не удастся получить комбинаторно полную порядка $k$ матрицу, о чем свидетельствует следующее утверждение. 
Утверждение 10. Для любых двоичных матрии, А и T, содержащих хотя бы два столбиа, матрица $B=T \otimes A \oplus \bar{T} \otimes \bar{A}$ не является комбинаторно полной порядка $k \geqslant 4$.

Доказательство. Из определения комбинаторно полных матриц нетрудно видеть, что если некоторая двоичная матрица не является комбинаторно полной порядка $k$, то она также не является комбинаторно полной порядка более $k$. Следовательно, достаточно доказать утверждение для $k=4$. Пусть матрица $T$ содержит $r$ строк. В терминах доказательства теоремы 1 рассмотрим первые два блок-столбца матрицы $B=T \otimes A \oplus \bar{T} \otimes \bar{A}$ (рис. 4 ).

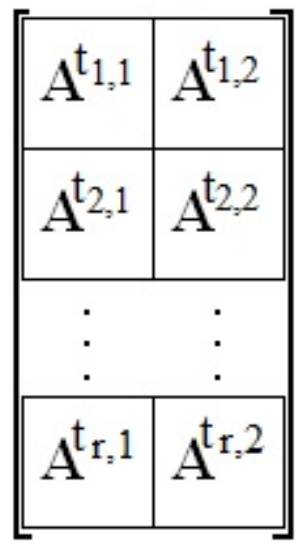

Рис. 4. Матрица $B$.

Рассмотрим подматрицу матрицы $B$, содержащую первые два столбца первого блок-столбца и первые два столбца второго блок-столбца. Нетрудно видеть, что в полученной подматрице матрицы $B$ содержатся такие строки длины 4 , что в каждой строке первые два элемента совпадают соответственно с третьим и четвертым или совпадают соответственно с инвертированным третьим и инвертированным четвертым элементами. Следовательно, данная подматрица не содержит следующих строк:

$$
(0001),(0010),(0100),(0111),(1000),(1011),(1101),(1110) .
$$

Таким образом, матрица $B$ не является комбинаторно полной порядка 4 , поэтому не является комбинаторно полной более высокого порядка.

\section{3. Построение комбинаторно полных матриц порядка 3 с малым отношением числа строк к числу столб- цов с помощью комбинаторно достаточных матриц}

Из теоремы 1 вытекает следующий алгоритм построения комбинаторно полных матриц порядка 3, который далее назовем алгоритмом тензорного расширения.

Bходные данные: комбинаторно достаточная порядка 3 матрица $T$ размера $r \times s$ и комбинаторно полная порядка 3 матрица $A_{0}$ размера $a \times b$. 
Алгоритм состоит в итеративном вычислении матриц $A_{i}, i=1,2, \ldots$, по формуле $A_{i}=T \otimes A_{i-1} \oplus \bar{T} \otimes \bar{A}_{i-1}$. Иными словами, на каждой итерации алгоритма тензорного расширения в матрице $T$ все единицы заменяются на $A_{i-1}$, а все нули на $\bar{A}_{i-1}$. Согласно теореме 1 матрица $A_{i}$ является комбинаторно полной порядка 3 и имеет размеры $\left(a \cdot r^{i}\right) \times\left(b \cdot s^{i}\right)$. Трудоемкость алгоритма сопоставима с размером итоговой матрицы $A_{i}$, то есть составляет порядка $a \cdot b \cdot(r \cdot s)^{i}$ битовых операций.

Далее опишем применение алгоритма тензорного расширения для поставленной задачи. Пусть $c>0$ - произвольное вещественное число. Предположим, что существует комбинаторно достаточная матрица $T$ с условием $r<s$. Тогда в построенном алгоритме для любого такого номера $i$, что $i>\log _{\frac{r}{s}}\left(\frac{b \cdot c}{a}\right)$, отношение числа строк матрицы $A_{i}$ к числу ее столбцов будет меньше $c$ :

$$
\frac{a \cdot r^{i}}{b \cdot s^{i}}<c
$$

Остается указать хотя бы одну комбинаторно достаточную матрицу порядка 3 , у которой число строк меньше числа столбцов. Поскольку условие комбинаторной достаточности более слабое по сравнению с условием комбинаторной полноты, такие матрицы нетрудно найти экспериментальным путем. Приведем примеры таких матриц.

$$
T_{1}=\left(\begin{array}{l}
0000000000 \\
0010000111 \\
0100000111 \\
1000000111 \\
0000011011 \\
0000101101 \\
0000110110 \\
0001001110 \\
0001010101
\end{array}\right)_{9 \times 10}, T_{2}=\left(\begin{array}{l}
000000000000 \\
001000011111 \\
010000011111 \\
100000100011 \\
000001100101 \\
000010101001 \\
000011110010 \\
011001101010 \\
000100101110 \\
011100110001
\end{array}\right)_{10 \times 12}
$$

Кроме того, из свойств комбинаторно достаточных матриц следует, что матрицы, полученные из $T_{1}$ и $T_{2}$ инвертированием и перестановкой строк и столбцов, также будут комбинаторно достаточными порядка 3.

Таким образом, доказано следующее утверждение.

Утверждение 11. Для любого сколь угодно малого вещественного значения $с>0$ при помощи алгоритма тензорного расширения можно построить бесконечно много комбинаторно полных матриц порядка 3 , у которых отношение числа строк $\kappa$ числу столби,ов меньше с.

Отметим одну особенность приведенного алгоритма. Оказывается, что даже если матрицы $A$ и $T$ не имеют одинаковых строк, матрица $B=T \otimes A \oplus \bar{T} \otimes \bar{A}$ может содержать одинаковые строки. Пусть матрица $A$ имеет размеры $m \times n$, а матрица $T$ имеет размеры $r \times s$. Проиндексируем строки матрицы $B$ парами чисел $(i, j)$, где $i \in\{1, \ldots, r\}$ обозначает номер блок-строки, $j \in\{1, \ldots, m\}$ - номер строки в блок-строке. Строки матрицы $T$ обозначим через $T_{i}^{\prime}, i \in\{1, \ldots, r\}$, строки матрицы $A$ через $A_{j}^{\prime}, j \in\{1, \ldots, m\}$. 
Утверждение 12. Для любых двоичных матрии, $A$ и $T$ в матрице $B=T \otimes A \oplus$ $\bar{T} \otimes \bar{A}$ строка с индексом $\left(i_{1}, j_{1}\right)$ совпадает со строкой с индексом $\left(i_{2}, j_{2}\right)$ тогда и только тогда, когда либо $A_{j_{1}}^{\prime}=A_{j_{2}}^{\prime}$ и $T_{i_{1}}^{\prime}=T_{i_{2}}^{\prime}$, либо $A_{j_{1}}^{\prime}=\overline{A_{j_{2}}^{\prime}}$ и $T_{i_{1}}^{\prime}=\overline{T_{i_{2}}^{\prime}}$.

Доказательство. Согласно введенным обозначениям в матрице $B$ строка с индексом $\left(i_{1}, j_{1}\right)$ имеет вид $\left(A_{j_{1}}^{\prime}{ }^{t_{i_{1}, 1}}, A_{j_{1}}^{\prime}{ }^{t_{i_{1}, 2}}, \ldots, A_{j_{1}}^{\prime}{ }^{t_{i_{1}, s}}\right)$, а строка с индексом $\left(i_{2}, j_{2}\right)$ имеет вид $\left(A_{j_{2}}^{\prime}{ }^{t_{i_{2}, 1}}, A_{j_{2}}^{\prime}{ }_{t_{2}, 2}, \ldots, A_{j_{2}}^{\prime}{ }^{t_{i_{2}, s}}\right)$. Равенство указанных строк эквивалентно совокупности равенств $A_{j_{1}}^{\prime}{ }^{t_{i_{1}, 1}}=A_{j_{2}}^{\prime}{ }^{t_{i_{2}, 1}}, A_{j_{1}}^{\prime{ }^{t_{i_{1}, 2}}}=A_{j_{2}}^{\prime}{ }^{t_{i_{2}, 2}}, \ldots, A_{j_{1}}^{\prime}{ }^{t_{i_{1}, s}}=A_{j_{2}}^{\prime}{ }^{t_{i_{2}, s}}$. Возможны только два варианта: либо строки $A_{j_{1}}^{\prime}$ и $A_{j_{2}}^{\prime}$ совпадают, и тогда $t_{i_{1}, v}=$ $t_{i_{2}, v}$ для всех $v \in\{1, \ldots, s\}$, то есть $T_{i_{1}}^{\prime}=T_{i_{2}}^{\prime} ;$ либо $A_{j_{1}}^{\prime}=\overline{A_{j_{2}}^{\prime}}$, и тогда $t_{i_{1}, v}=t_{i_{2}, v} \oplus 1$, то есть $T_{i_{1}}^{\prime}=\overline{T_{i_{2}}^{\prime}}$.

Поскольку целью алгоритма является построение комбинаторно полных матриц, у которых число строк меньше числа столбцов, то при возникновении повторов строк можно просто удалить одинаковые строки из матрицы $B$, и тем самым дополнительно уменьшить число строк.

\section{Список литературы}

1. Голощапов В.Н., Ролдугин П.В., "Оценка числа переменных булевых функций небольшого веса, не содержащих имплицент меньшей длины”, Дискретная математика, 25:1 (2013), 45-62.

2. Голощапов В.Н., Ролдугин П.В., "Построение булевых функций, не имеющих имплицент от малого числа переменных", в печати, Вестник Московского государственного университета леса «Лесной вестник».

3. Ролдугин П.В., Тарасов А.В., "О булевых функциях без верхних биюнктивных аналогов. Математические вопросы криптографии”, 4:1 (2013), 123-140.

4. Ролдугин П.В., Тарасов А.В., “Функции без коротких имплицент. Часть I: нижние оценки весов", в печати, Математические вопросы криптографии.

5. Ролдугин П.В., Тарасов А.В., "Функции без коротких имплицент. Часть II: методы построения", в печати, Математические вопросы криптографии.

6. Самофалов К.Г., Романкевич А.М., Валуйский В.Н., Каневский Ю.С., Пиневич М.М., Прикладная теория иифровых автоматов, К.: Вища школа, 1987.

7. Воеводин В.В., Кузнецов Ю.А., Матрицы и въчисления, М.: Наука. ГРФМЛ, 1984. 